\title{
Exponentially Small Splittings of Separatrices with applications to KAM Theory and Degenerate Bifurcations
}

\author{
Philip Holmes, Jerrold Marsden, and Jurgen Scheurle \\ $2 / 87,6 / 87,10 / 88$
}

\section{Abstract}

Both upper and lower estimates are established for the separatrix splitting of rapidly forced systems with a homoclinic orbit. The general theory is applied to the equation

$$
\ddot{\varphi}+\sin \varphi=\delta \sin \left(\frac{t}{\varepsilon}\right)
$$

for illustration. There are two types of results. First, fix $\eta>0$ and let $0<\varepsilon \leq 1$ and $0 \leq \delta \leq \delta_{0}$ where $\delta_{0}$ is sufficiently small. If the separatrices split, they do so by an amount that is no more than

$$
c \delta \exp \left(-\frac{1}{\varepsilon}\left(\frac{\pi}{2}-\eta\right)\right)
$$

where $\mathrm{C}=\mathrm{C}\left(\delta_{0}\right)$ is a constant depending on $\delta_{0}$ but is uniform in $\varepsilon$ and $\delta$. Second, if we replace $\delta$ by $\varepsilon^{p} \delta, p \geq 8$, then we have the sharper estimate

$$
C_{2} \varepsilon^{p} \delta e^{-\pi / 2 \varepsilon} \leq \text { splitting distance } \leq C_{1} \varepsilon^{p} \delta e^{-\pi / 2 \varepsilon}
$$

for positive constants $\mathrm{C}_{1}$ and $\mathrm{C}_{2}$ depending on $\delta_{0}$ alone. In particular, in this second case, the Melnikov criterion correctly predicts exponentially small splitting and transversal intersection of the separatrices. After developing this theory we discuss some of its applications, concentrating on a 2:I resonance that occurs in a KAM (Kolmogorov, Arnold, and Moser) situation and in the forced saddle node bifurcation described by

$$
\ddot{x}+\mu x+x^{2}+x^{3}=\delta f(t) .
$$




\section{Introduction}

In Poincare's celebrated memoir [1890] on the 3-body problem, he introduced the mechanism of transversal intersection of separatrices which obstructs the integrability of the equations and the attendant convergence of series expansions for the solutions. This idea has been developed by Birkhoff and Smale using the horseshoe construction to describe the resulting chaotic dynamics. However, in the region of phase space studied by Poincare, it has never been proved (except in some generic sense that is not easy to interpret in specific cases) that the equations really are nonintegrable. In fact Poincare himself traced the difficulty to the presence of terms in the separatrix splitting which are exponentially small. A crucial component of the measure of the splitting is given by the following formula of Poincare [1890, page 223]:

$$
J=\frac{-8 \pi i}{\exp \left(\frac{\pi}{\sqrt{2 \mu}}\right)+\exp \left(-\frac{\pi}{\sqrt{2 \mu}}\right)}
$$

which is exponentially small (or beyond all orders) in $\mu$. Poincare was well aware of the difficulties that this exponentially small behavior causes; on page 224 of his article, he comments that "En d'autres termes, si on regarde $\mu$ comme un infiniment petit du premier ordre, la distance BB', sans ètre nulle, est un infiniment petit d'ordre infini. C'est ainsi que la fonction $\mathrm{e}^{-1 / \mu}$ est un infiniment petit d'ordre infini sans ètre nulle .... Dans l'example particulier que nous avons traité plus haut, la distance $\mathrm{BB}$ ' est du mème ordre de grandeur que l'integral $\mathrm{J}$, c'est à dire que $\exp (-\pi / \sqrt{2 \mu})$."

In this paper we overcome some of the essential difficulties that are encountered in this type of problem, in KAM theory, and in chaotic motions occurring in the unfoldings of degenerate singularities. Based on numerical evidence and formal calculations, it is known that one should get exponentially fine splittings and exponentially long escape times for problems of this type. Some rigorous but rough upper bounds for this phenomena have been given by Nekhoroshev [1971,77] and Neishtadt [1984]; see also the discussion in Arnold [1978], p.395ff and 407, Chirikov [1979] and Simo and Fontich [1985]. The analyticity argument of Cushman [1978] and Kozlov [1984] (and reference therein) uses the Poincare-Melnikov method to prove that the separatrices do split for most parameter values. However, it is not easy to prove from these arguments that splittings really do occur for specific parameter values and what the sharp upper and lower estimates for the splitting distances are. The seriousness and significance of this difficulty was further emphasized by Sanders [1982]. 
In KAM theory one also finds that the splitting of separatrices is governed by systems of the form considered here, and so would be formally beyond all orders if a power series in the perturbation parameter were developed. Indeed, a formal calculation based on the Melnikov method shows that the splitting of separatrices is probably of exponentially small order, a phenomenon discussed in Amold's book (see especially page 397). Zehnder [1973] also shows that there are transverse homoclinic orbits for generic nonlinearities in KAM theory. In a similar fashion, the same type of behavior arises in the unfolding of degenerate singularities, such as the interaction of the Hopf and the pitchfork or transcritical bifurcation (see Guckenheimer and Holmes [1983] and Scheurle and Marsden [1984] for discussions of this bifurcation and for further references). See also the paper of Dangelmayr and Knobloch [1987] for the case of symmetry breaking bifurcations and the work of Golubitsky and Stuart [1986] for the application of unfolding techniques to the Taylor Couette problem, where it is expected that similar phenomena will occur. Since these splittings are exponentially small, standard methods for detecting them based on averaging, normal forms, or perturbation expansions using power series in $\varepsilon$, will not succeed. This is also behind the fact that one has, in general, divergence of the Birkhoff series.

In this paper, we give a new method that overcomes many of these difficulties. We give sharp upper bounds, with the constant in the exponential being the distance of the nearest pole in the complex t-plane of the unperturbed homoclinic orbit to the real axis. If a high enough power of $\varepsilon$ is present in front of the forcing term then there is a lower bound for the splitting, which is also exponentially small with the same exponential factor. In the latter case, the Melnikov integral is sufficient to predict the transversality of, and to estimate the magnitude of the splitting. In general, however, it appears that one must go to higher orders to obtain a predictable criterion, in which case one has to revert to an intricate calculation, or else use the Cushman-Koslov analyticity argument, which only gives a generic result.

Our approach is based on a convergent iteration scheme using the Liapunov-Perron method and a special extension of the scheme to the complex $t$ plane that enables us to estimate the splitting distance. A naive extension will run into difficulties since the forcing term $\sin (t / \varepsilon)$ is exponentially big for $t$ in a complex strip. As mentioned above, these estimates relate the singularities in the complex plane and the factor in the exponential [the separatrix for the homoclinic orbit in the pendulum case has one component given by sech $t$, which has simple poles at $t= \pm i \pi / 2$, and the corresponding exponential factor is $\exp (-\pi / 2 \varepsilon)$.] Because of this, one can conjecture a connection between the results here and the Painlévé property. The work of Ziglin [1982], van Moerbeke [1983] and Bountis et.al.[1986] may be helpful in this regard. The key to our method is that the special iteration scheme preserves the exponentially small structure, with the same factor in the exponent at each stage, controls the possible accumulation in the pole behavior, and exhibits the cancellation of terms that move each of the stable and unstable manifolds (and the 
hyperpolic fixed point) an amount that is algebraic in $\varepsilon$, even though the difference between them is exponentially small. The key points of the proof are given here; a more detailed paper is in preparation.

There have been other approaches to exponentially small phenomena bäsed on asymptotic methods. For example, the works of Meyer [1976] on adiabatic variation, Meyer [1982] on wave reflection and quasiresonance, Segur and Kruskal [1987] on breathers in the $\varphi^{4}$ model, and Kruskal and Segur [1987] on dendritic crystals, use this technique. While there seem to be some points in common with our approach, it appears that additional work would be needed to apply and justify the estimates that we obtain for separatrix splitting.

Acknowledgements We thank B. Birnir, J. Carr, B. Chirikov, W. Eckhaus, M. Golubitsky, J. Guckenheimer, K. Kirchgassner, M. Kummer, R. Meyer, and H. Segur for useful comments. The research of P. Holmes was partially supported by NSF grant MSM 84-02069 and AFOSR contract 84-0051, and that of J. Marsden and J. Scheurle was partially supported by NSF grant DMS 87-02502 and DOE contract DE-AT03-85ER12097.

\section{$\$ 1$ Preliminaries}

We begin by recalling a few basic facts about the standard Poincare - Melnikov method. The phase portrait of the simple pendulum

$$
\ddot{\phi}+\sin \phi=0
$$

is as shown in Figure 1 in the $(\phi, v)$ plane, where $v=d \phi / d t$. The homoclinic orbits shown there are explicitly given by the solutions

$$
\left.\begin{array}{l}
\bar{\phi}(t)= \pm 2 \tan ^{-1}(\sinh t) \\
\bar{v}(t)= \pm 2 \operatorname{sech}(t)
\end{array}\right\} .
$$

We observe for later use that sech $t$ has poles in the complex $t$-plane at $t= \pm i \pi / 2$. 


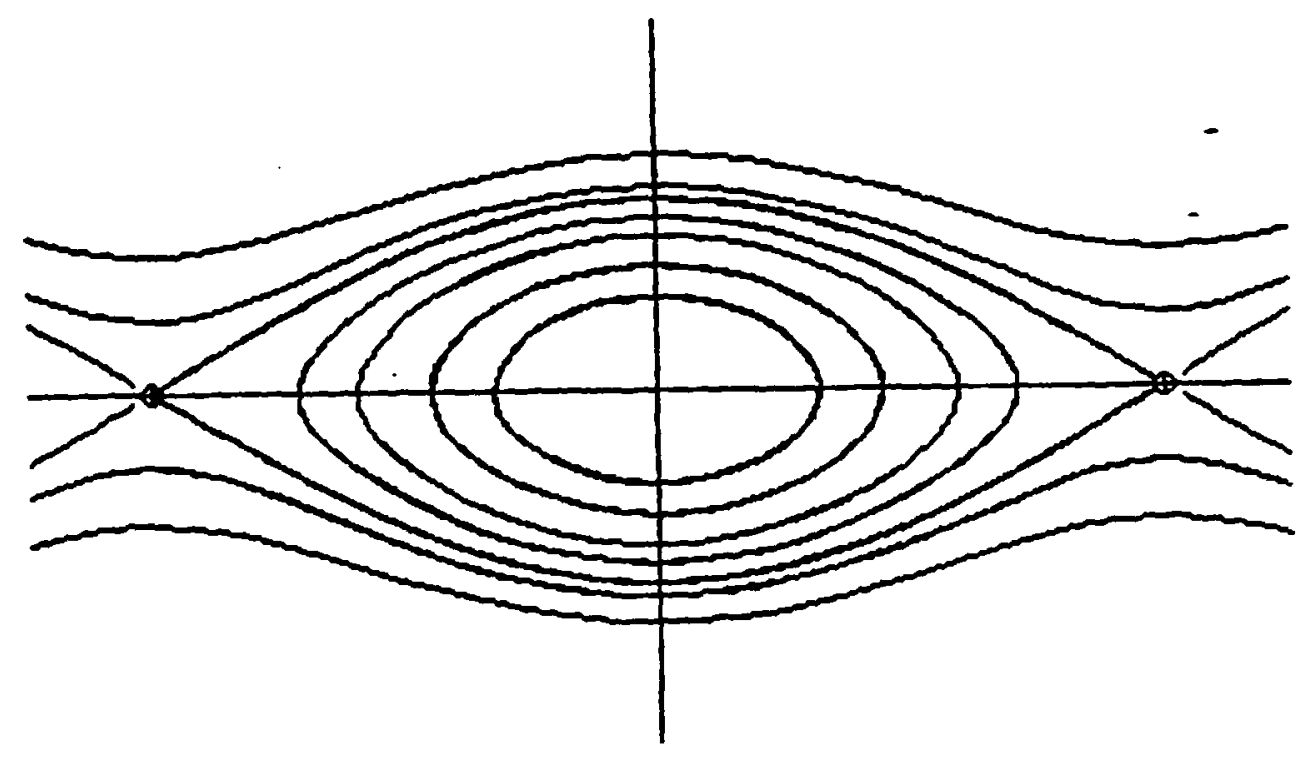

Figure 1. Phase portrait of the simple pendulum.

If we modify (1) by including a T-periodic forcing, we get the equation

$$
\ddot{\phi}+\sin \phi=\varepsilon f(t),
$$

for which the dynamics is conveniently described by the Poincare map $P\left(t_{0}\right): \mathbb{R}^{2} \rightarrow \mathbb{R}^{2}$ defined by mapping initial conditions $\left(\phi_{0}, v_{0}\right)$ at time $t_{0}$ to the solution after one period, at time $t_{0}+T$. For small $\varepsilon$, the hyperbolic fixed points for (1.1) get perturbed to fixed points for $P\left(t_{0}\right)$ (i.e., periodic orbits for (1.3)) and $P\left(t_{0}\right)$ has stable and unstable manifolds at these fixed points which, in general, intersect. This leads one to define the splitting distance

$$
d=\max _{b} d\left(t_{0}\right)
$$

and the splitting angle

$$
\alpha=\max _{b} \alpha\left(t_{0}\right)
$$

where, for any $t_{0}, d\left(t_{0}\right)$ and $\alpha\left(t_{0}\right)$ are shown in Figure 2 . 


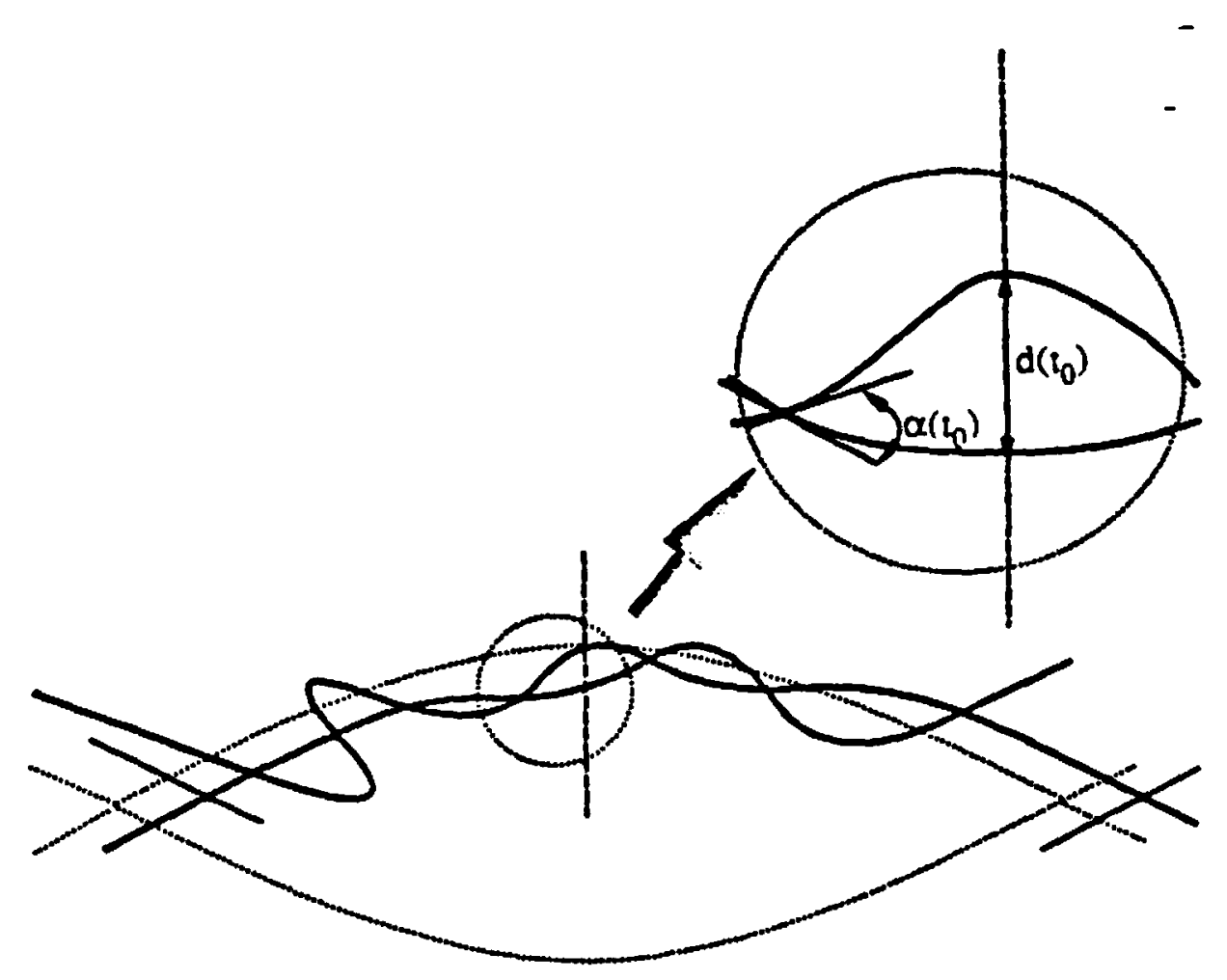

Figure 2. The splitting distance and angle.

This splitting distance and angle are correlated with the thickness of the stochastic layer; the trajectories of some sample points are shown in Figure 3 for illustration. One should be cautious, however, that there is little analytic work on the precise relation between the splitting distance and angle and the thickness of the stochastic layer. However, the celebrated horseshoe construction of Poincare, Birkhoff, and Smale does establish that a transversal intersections ( $\alpha \neq$ 0 ) implies the existence of complicated orbits (and periodic orbits with arbitrarily high period) and thus warrants using the word "chaotic" to describe the dynamics. 


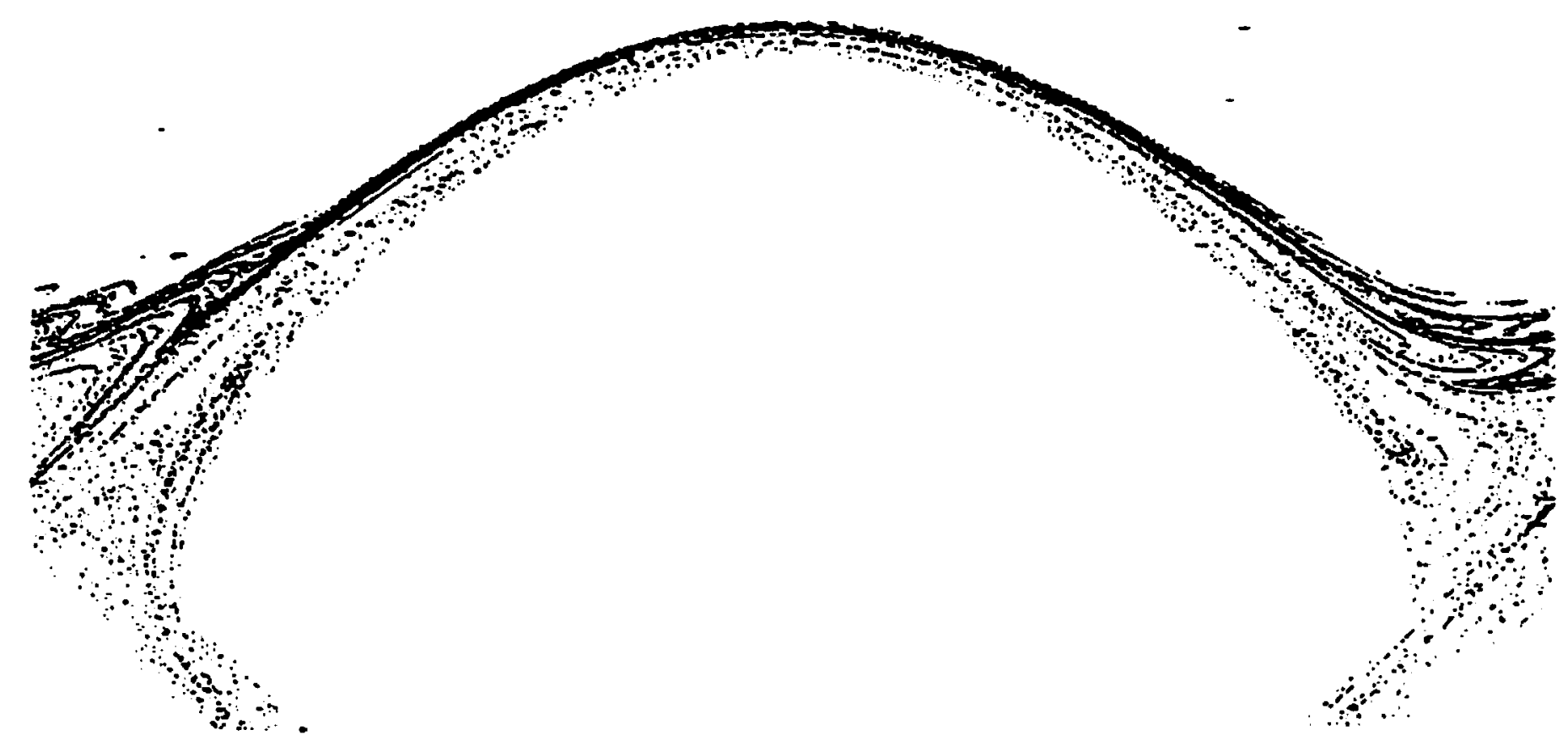

Figure 3. Orbits of points under the Poincare map of (1.3) for $f(t)=(0.1) \sin t$ (plot courtesy of B. Bimir).

The splitting distance is typically measured by a Poincare-Melnikov function. For a planar Hamiltonian system

$$
\left.\begin{array}{l}
\dot{q}=\frac{\partial H}{\partial p}+\varepsilon \frac{\partial K}{\partial p} \\
\dot{p}=-\frac{\partial H}{\partial q}-\varepsilon \frac{\partial K}{\partial q}
\end{array}\right\},
$$

where $K=K(q, p, t)$ is a perturbing T-periodic Hamiltonian, the Poincaré - Melnikov function is the $T$-periodic function

$$
M\left(t_{0}\right)=\int_{-\infty}^{\infty}\{H, K\}\left(\bar{q}(t), \bar{p}(t), t+t_{0}\right) d t
$$

the splitting distance is proportional to 


$$
d=\varepsilon \max _{0}\left|M\left(t_{0}\right)\right|+O\left(\varepsilon^{2}\right),
$$

and the angle is proportional to

$$
\alpha=\varepsilon \max _{b}\left|M^{\prime}\left(t_{0}\right)\right|+O\left(\varepsilon^{2}\right)
$$

This follows readily from an analysis of the first variation equation. See, for instance, Holmes and Marsden [1982] and Guckenheimer and Holmes [1983] for discussions and proofs. For example, for

$$
\ddot{\phi}+\sin \phi=\varepsilon \sin \omega t
$$

one finds

$$
M\left(t_{0}\right)=2 \pi \operatorname{sech}\left(\frac{\pi \omega}{2}\right) \cos \left(\omega t_{0}\right)
$$

by evaluating (1.7) using residues, noting the pole of sech $t$ at $i \pi / 2$. Thus,

$$
d=2 \pi \varepsilon \operatorname{sech}\left(\frac{\pi \omega}{2}\right) \quad \text { and } \quad \alpha=2 \pi \omega \varepsilon \operatorname{sech}\left(\frac{\pi \omega}{2}\right)
$$

\section{Exponentially Small Splittings}

To illustrate the main idea, first consider the rapidly forced pendulum

$$
\ddot{\phi}+\sin \phi=\varepsilon \sin (t / \varepsilon)
$$

If one applies equation (1.12), one finds the splitting distance should be of the order

$$
d=2 \pi \varepsilon e^{-\pi / 2 \varepsilon} .
$$


(The constant $2 \pi$ is not important -- it depends on the units of measure; for example, it may be convenient to use the unperturbed energy as a distance measure.) However, (2.2) is not easy to justify; for one thing, the errors in (1.8) are $O\left(E^{2}\right)$, while (2.2) is already smaller than any power of $\varepsilon$.

There are two main results for problems of this sort as follows:

\section{UPPER ESTIMATE Consider}

$$
\ddot{\phi}+\sin \phi=\delta \sin (t / \varepsilon) \text {. }
$$

For any $\eta>0$ there is $a \delta_{0}>0$ and a constant $C=C\left(\eta, \delta_{0}\right)$ such that, for all $\varepsilon$ and $\delta$ satisfying $0<\varepsilon \leq 1$ and $0<\delta \leq \delta_{0}$, we have

$$
\text { splitting distance } \leq \operatorname{Cox} \exp \left[-\left(\frac{\pi}{2}-\eta\right) \frac{1}{\varepsilon}\right] \text {. }
$$

There is a similar estimate for the splitting angle.

\section{LOWER ESTIMATE AND SHARP UPPER ESTIMATE Consider}

$$
\ddot{\phi}+\sin \phi=\varepsilon^{p} \delta \sin (t / \varepsilon)
$$

If $\mathrm{p} \geq 8$, then there is $a \delta_{0}>0$ and (absolute) constants $C_{1}$ and $C_{2}$ such that, for all $\varepsilon, \delta$ satisfying $0<\varepsilon \leq 1$ and $0<\delta \leq \delta_{0}$, we have

$$
C_{2} \varepsilon^{p} \delta e^{-\pi / 2 \varepsilon} \leq \text { Splitting Distance } \leq C_{1} \varepsilon^{p} \delta e^{-\pi / 2 \varepsilon}
$$

Observe that $\pi / 2$, which appears in the exponent in both estimates, is the distance from the real axis to the closest pole of sech $t$; see Figure 4. 


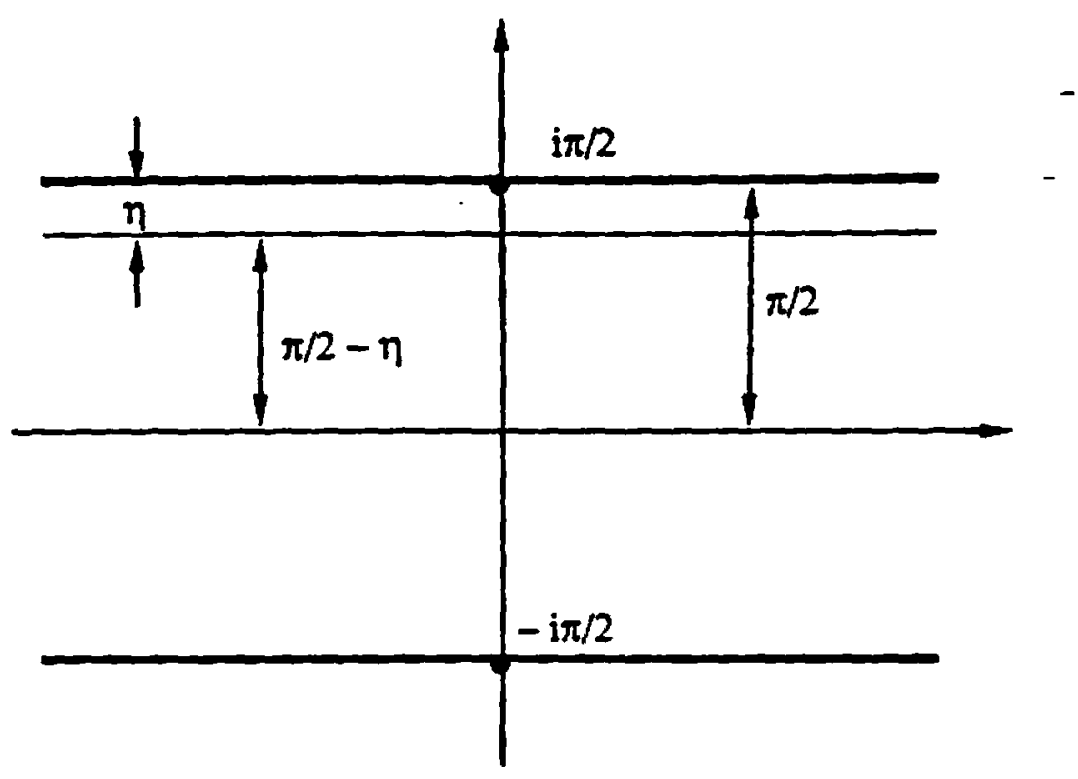

Figure 4. The exponent in the exponential estimate is the distance to the nearest pole of the homoclinic orbit in the complex t-plane.

These estimates are special cases of estimates for a planar system

$$
\dot{u}=g(u, \varepsilon)+\varepsilon^{p} \delta h\left(u, \varepsilon, \frac{t}{\varepsilon}\right)
$$

where one assumes:

- $g$ and $h$ are entire in $u$ and $\varepsilon$;

- $\mathrm{h}$ is of Sobolev class $\mathrm{H}^{1}$ (for the splitting distance results) or $\mathrm{H}^{2}$ (for the splitting angle results) and T-periodic in the variable $\theta=t / E$;

- $\dot{u}=g(u, \varepsilon)$ has a homoclinic orbit $\bar{u}(\varepsilon, t)$ analytic in $t$ on a strip in the complex $\mathrm{t}$ - plane, with width $\mathrm{r}$.

under additional assumptions on the fundamental solution of the first variation equation

$$
\dot{v}=D_{u} g(u, \varepsilon) \cdot v
$$


which can be checked to hold in the pendulum example, there are analogues of the upper and lower estimates above for this general situation, with $\pi / 2$ replaced by $r$. We shall give additional details in the subsequent sections. The proofs depend on detailed estimates of the terins in an iterative process in the complex strip that are used to define the invariant manifolds. It is important to extend these iterates to the complex strip in the proper way; as we have mentioned, $\sin (t / E)$ becomes very large for complex $t$ and naively extended iteration procedures for the stable and unstable manifolds will lead to unbounded sequences of functions.

\section{The Hypotheses and Set-Up.}

We recall some of the general theory and the ideas involved in the proofs from Holmes, Marsden and Scheurle [1988] for the convenience of the reader. We consider a differential equation of the following form

$$
\dot{u}=g(u, \varepsilon)+\varepsilon^{p} \delta h\left(u, \varepsilon, \frac{t}{\varepsilon}\right)
$$

where $p$ is a positive integer (one can think of the term $\varepsilon^{p}$ as being part of $h$ or as being divided between $\delta$ and $h$ as is appropriate), $u=(x, y) \in \mathbb{R}^{2}, \varepsilon>0, g(u, \varepsilon): \mathbb{C}^{2} \times \mathbb{C} \rightarrow \mathbb{C}^{2}$ is entire, $h$ is entire in $(u, \varepsilon)$ and is $2 \pi$-periodic and $C^{l}$ (or of Sobolev class $H^{l}$ ) in its third arguement $t / \varepsilon$. Both $g$ and $h$ are assumed to be real for real values of their arguments. (The $\mathrm{H}^{1}$ assumption on $h$ is needed below to get bounds on the splitting distance; for exponentially small bounds on the

angle at a transversal intersection, we need to assume that $h$ is of class $H^{2}$ in $t / \varepsilon$ - see Remark 1 at the end of section 5.)

Although it is not really needed, we shall introduce a symmetry condition for simplicity. (A more general case without this condition is discussed at the end of this paper.) Namely, we assume that the system (2.1) is reversible in the sense that there is a real linear reflection operator $R: \mathbb{R}^{2} \rightarrow \mathbb{R}^{2}$ i.e. a $2 \times 2$ matrix satisfying $R^{2}=$ Identity, with eigenvalues \pm 1 , and satisfying the following conditions:

$$
g(R u, \varepsilon)=-R g(u, \varepsilon) \quad \text { and } h(R u, \varepsilon,-t / \varepsilon)=-\operatorname{Rh}(u, \varepsilon, t / \varepsilon) \text {. }
$$

For instance, for the example given in the preceding section, we take

$$
g(x, y)=(y,-\sin x), \quad h((x, y), \varepsilon, t / \varepsilon)=(0, \sin (t / \varepsilon)) \quad \text { and } \quad R(x, y)=(-x, y)
$$


Assume that the homogeneous equation $\mathrm{u}=\mathrm{g}(u, \varepsilon)$ has a homoclinic orbit $\Gamma_{\varepsilon}$ which is asymptotic to a hyperbolic fixed point; we write the homoclinic orbit as $u=\bar{u}_{\varepsilon}(t)$. We shall assume that $\bar{u}_{\varepsilon}(t)$ has an analytic continuation in the complex $t$ plane into a complex strip $S_{\varepsilon}$ defined to be the set of complex numbers $z$ such that $|\operatorname{Im} z| \leq r_{\varepsilon}$, where $r_{\varepsilon}$ is some positive real number. Typically, $\vec{u}_{\varepsilon}(t)$ will be an analytic function in $t$, and will be analytic in a strip, with $r_{\varepsilon}$ smaller than the smallest distance of the poles to the real axis. We assume that the initial condition of the homoclinic orbit satisfies $R \bar{u}_{\varepsilon}(0)=\bar{u}_{\varepsilon}(0)$, so that $R \bar{u}_{\varepsilon}(-t)=\bar{u}_{\varepsilon}(t)$. In the example, the homoclinic orbit is given by

$$
(\bar{\varphi}(t), \zeta(t))=2\left(\tan ^{-1}(\sinh t), \operatorname{sech} t\right)
$$

where $\zeta=\mathrm{d} \varphi / \mathrm{dt}$, so this assumption is clear.

As indicated in section 2, there are two cases to consider. In the first, we choose $\mathrm{p}=0$ and $r_{\varepsilon}=(\pi / 2)-\eta$ for a fixed $\eta>0$ and in the second, we choose $r_{\varepsilon}=(\pi / 2)-\varepsilon$ (and later we will require $p \geq 8$ ).

The first variation equation

$$
\dot{v}=D_{u} g\left(\bar{u}_{\varepsilon}(t), \varepsilon\right) \cdot v
$$

has exponential dichotomies corresponding to $t$ in $\mathbb{R}^{+}$and $\mathbb{R}^{-}$(see for example, Hartman [1982], Ch. 13). That is, the plane splits into two subbundles

$$
\mathbb{R}^{2}=\mathbf{X}_{t, \varepsilon} \oplus \mathbf{Y}_{t, \varepsilon}
$$

that are invariant under the evolution of the first variation equation, such that the components $\varphi_{1}$ and $\varphi_{2}$ of the fundamental solution matrix $\varphi(t, \tau)$, which are defined by restriction of $\varphi$ to $X_{\tau, \varepsilon}$ and $Y_{\tau, \varepsilon}$ respectively, satisfy the inequalities:

$$
\begin{array}{ll}
\left|\varphi_{1}(t, \tau) \xi\right| \leq \mathrm{Ke}^{-\alpha(\tau-\tau)}|\xi| & \text { (if } \tau \geq \tau) \\
\left|\varphi_{2}(t, \tau) \eta\right| \leq \mathrm{Ke}^{-\alpha(t-\tau)}|\eta| & \text { (if } \tau \leq \tau)
\end{array}
$$


for $t, \tau$ satisfying $-\infty<1, \tau \leq 0$, and where $\xi \in X_{\tau, \varepsilon}$, and $\eta \in Y_{\tau, \varepsilon}$. For simplicity of notation, we have suppressed the possible $\varepsilon$ dependence of $\varphi, \varphi_{1}$, and $\varphi_{2}$. Similarly,

$$
\begin{array}{ll}
\left|\varphi_{1}(t, \tau) \xi\right| \leq K \mathrm{e}^{\alpha(\tau-t)}|\xi| & \text { (if } \tau \leq t)- \\
\left|\varphi_{2}(t, \tau) \eta\right| \leq K \mathrm{e}^{\alpha(t-\tau)}|\eta| & \text { (if } \tau \geq \mathrm{t})
\end{array}
$$

for $t, \tau$ satisfying $0 \leq \tau, t<\infty$, and where $\xi \in X_{\tau, \varepsilon}$ and $\eta \in Y_{\tau, \varepsilon}$. In these equations $\alpha$ and $K$ are positive constants. The constant $\alpha$ is related to the eigenvalues of the linearization of the equation at the hyperbolic fixed point. We choose the dichotomies amongst all possible ones by the requirement that at $t=0$, the bundles satisfy

$$
\begin{aligned}
& X_{0, \varepsilon} \text { is the eigenspace of } R \text { corresponding to the eigenvalue }-1 \\
& Y_{0, \varepsilon} \text { is the eigenspace of } R \text { corresponding to the eigenvalue } 1
\end{aligned}
$$

In the example, we take the bundles to be the tangential space to the homoclinic orbit and the normal direction at the point $t=0$ swept out by the flow of the first variation equation. The first variation solution is explicitly found in this case to be as follows

$\varphi_{1}(t, \tau) \xi=\frac{1}{2}[\{\cosh \tau+\operatorname{sech} \tau-\tau \operatorname{sech} \tau \tanh \tau\} \psi-\{\sinh \tau+\tau \operatorname{sech} \tau\} v]\left(\begin{array}{c}\operatorname{sech} t \\ -\operatorname{sech} t \tanh t\end{array}\right)$

$\Phi_{2}(\mathfrak{t}, \tau) \eta=\frac{1}{2}[\{\operatorname{sech} \tau \tanh \tau\} \Psi-\{\operatorname{sech} \tau\} v]\left(\begin{array}{c}\sinh t+t \operatorname{sech} t \\ \cosh t+\operatorname{sech} t-t \operatorname{sech} t \tanh t\end{array}\right)$

where $v$ has components $(\psi, v)$ in the original coordinate system and where $\xi$ and $\eta$ are the projections of $v$ onto the spaces $X_{\tau, \varepsilon}$ and $Y_{\tau, \varepsilon}$ respectively.

\section{$\$ 4$ The Iteration Method.}

We shall locate the stable and unstable manifolds of the perturbed equation using a special Liapunov-Perron type iteration scheme that is coupled with a Fourier expansion and a certain extension to the complex $t$-plane. It will be important to keep track of the estimates during the 
iteration process itself. We write the perturbed stable and unstable manifold of the hyperbolic fixed point as follows:

$$
u^{ \pm}\left(t, t_{0}, \varepsilon, \delta\right)=\bar{u}_{\varepsilon}\left(t-t_{0}\right)+\delta v^{ \pm}\left(t-t_{0}, t_{0}, \varepsilon, \delta\right)
$$

where $t \geq t_{0}$ in the + case, and $t \leq t_{0}$ in the - case. Dropping the \pm and writing $s=t-t_{0}$, we get an equation for $v$, regarded as a function of $s, b_{0}, \varepsilon$ and $\delta$ by substituting (3.1) into (2.1); the stable and unstable manifolds will later be picked out by looking for bounded solutions of the resulting fixed point problem. We first compute (suppressing the $\varepsilon$ and $\delta$ dependence for the moment):

$$
\dot{v}-A(s) v=F\left(v, s,\left(s+t_{0}\right) / \varepsilon\right)
$$

where

$$
A(s)=D_{u} g\left(\bar{u}_{\varepsilon}(s), \varepsilon\right)
$$

and

$$
\begin{aligned}
& f\left(v, s, \frac{s+t_{0}}{\varepsilon}\right)= \\
& \quad \frac{1}{\delta}\left[g\left(\bar{u}_{\varepsilon}(s)+\delta v, \varepsilon\right)-g\left(\bar{u}_{\varepsilon}(s), \varepsilon\right)-A(s) \delta v\right]+\varepsilon^{p} h\left(\bar{u}_{\varepsilon}(s)+\delta v, \varepsilon, \frac{s+t_{0}}{\varepsilon}\right)
\end{aligned}
$$

Here the $\varepsilon^{p}$ is grouped with $h$, but we also could group appropriate powers with $\delta$; this freedom is important later. We look for solutions of (4.2) that are uniformly bounded in the \pm cases by reformulating it as a fixed point problem for the following integral equation

$$
v=K^{ \pm} F\left(v, s, \frac{s+t_{0}}{\varepsilon}\right)
$$

where $\mathrm{K}^{ \pm}$are the linear operators (again with the $\varepsilon$ dependence supressed) that are given by 


$$
\begin{aligned}
& \left(K^{+} f(s)=\int_{0}^{s} \varphi_{1}(s, \sigma) f_{1}(\sigma) d \sigma-\int_{s}^{\infty} \varphi_{2}(s, \sigma) f_{2}(\sigma) d \sigma \quad \text { for all } s \geq 0\right. \\
& \left(K^{-} f\right)(s)=\int_{0}^{s} \varphi_{1}(s, \sigma) f_{1}(\sigma) d \sigma+\int_{-\infty}^{s} \varphi_{2}(s, \sigma) f_{2}(\sigma) d \sigma \quad \text { for all } s \leq 0
\end{aligned}
$$

Here the projections of $f$ onto the dichotomy subspaces (2.4) are denoted $f_{1}$ and $f_{2}$. With $K^{ \pm}$ thought of as operating on the space of bounded continuous (vector valued) functions on $\mathbb{R}^{ \pm}$, one gets a contraction mapping for sufficiently small $\delta_{0}$ and a unique fixed point that can be solved for iteratively (see, for example, Harman [1982], Chapter 12, part III). The stable and unstable manifolds are determined by the fixed points, which we denote by $v^{+}\left(s, t_{0}\right)$ and $v\left(s, t_{0}\right)$, where we again suppress the dependence on $\varepsilon$ and $\delta$. We start the iteration scheme in each case with the zero solution and then define inductively

$$
{ }^{(n+1)} v^{ \pm}\left(s, t_{0}\right)=K^{ \pm} F\left(v^{(n)}\left(s, t_{0}\right), s, \frac{s+t_{0}}{\varepsilon}\right)
$$

so that $(n)_{v} \pm\left(s, t_{0}\right)$ converges to $v^{ \pm}\left(s, t_{0}\right)$ as $n \rightarrow \infty$.

\section{$\$ 5$ Estimates for the Splitting Distance}

The next step is to estimate the splitting distance between the stable and unstable manifolds. To do this we estimate the following quantity:

$$
\Delta\left(t_{0}, \varepsilon, \delta\right)=\left|u^{+}\left(t_{0}, t_{0}, \varepsilon, \delta\right)-u^{-}\left(t_{0}, t_{0}, \varepsilon, \delta\right)\right|=\delta\left|v^{+}\left(0, t_{0}, \varepsilon, \delta\right)-v^{-}\left(0, t_{0}, \varepsilon, \delta\right)\right|
$$

where $u^{ \pm}\left(s+t_{0}, t_{0}, \varepsilon, \delta\right)=\bar{u}_{\varepsilon}(s)+\delta v^{ \pm}\left(s, t_{0}, \varepsilon, \delta\right)$ are the stable and unstable manifolds of the perturbed equation. The splitting distance is defined to be the maximum of $\Delta\left(t_{0}, \varepsilon, \delta\right)$ over one $2 \pi \varepsilon$ period in $t_{0}$. We extend each of the solutions of the iteration scheme (4.7) to strips in the complex $t$ plane. We do this in a way that makes the iterates uniformly bounded in the appropriate ( $\varepsilon$ dependent) half strips

$$
S^{+}=\left\{z \in \mathbb{C}|| \operatorname{Im} z \mid \leq r_{\varepsilon} \text { and } \operatorname{Re} z \geq 0\right\}
$$




$$
S-=\left\{z \in C|| \operatorname{Im} z \mid \leq r_{\varepsilon} \text { and } \operatorname{Re} z \leq 0\right\}
$$

and the vertical segment

$$
S^{0}=\left\{z \in \mathbb{C}|| \operatorname{Im} z \mid \leq r_{\varepsilon} \text { and } \operatorname{Re} z=0\right\} \text {. }
$$

Thus, a uniform exponential estimate on the distance between the iterates

$$
{ }^{(n)} \Delta\left(t_{0}, \varepsilon, \delta\right)=\left.\delta\right|^{(n)} v^{+}\left(0, t_{0}, \varepsilon, \delta\right)-{ }^{(n)} v^{-}\left(0, t_{0}, \varepsilon, \delta\right) \mid
$$

produces the corresponding result for the limiting solution as $\mathbf{n} \rightarrow \infty$.

Let $\theta=\left(s+t_{0}\right) / \varepsilon$ and consider the following iteration scheme for an ( $\varepsilon$ and $\delta$ dependent) function $w(s, \theta)$ :

$$
(n+1) w^{ \pm}(s, \theta)=L^{ \pm} F\left({ }^{(n)} w^{ \pm}(s, \theta), s, \theta\right)
$$

where we start with $w=0$, and define $L^{ \pm}$as follows. For any vector valued function

$$
f(s, \theta)=\sum_{k=-\infty}^{\infty} f_{k}(s) e^{i k \theta}, \text { where } s \in S^{ \pm} \text {and } \theta \in \mathbb{R} \text {, }
$$

we set

$$
L^{ \pm} f=\sum_{k=-\infty}^{\infty} a_{k}^{ \pm}(s) e^{i k \theta}
$$

where we define

$$
\begin{aligned}
& a_{0}^{+}(s)=\int_{0}^{s} \varphi_{1}(s, \sigma) f_{0,1}(\sigma) d \sigma-\int_{s}^{\infty} \varphi_{2}(s, \sigma) f_{0,2}(\sigma) d \sigma \\
& a_{0}^{-}(s)=\int_{0}^{s} \varphi_{1}(s, \sigma) f_{0,1}(\sigma) d \sigma+\int_{-\infty}^{s} \varphi_{2}(s, \sigma) f_{0,1}(\sigma) d \sigma
\end{aligned}
$$




$$
\mathrm{a}_{k \mathrm{k}}^{+}(s)=\int_{ \pm \mathrm{i} \varepsilon}^{s} \varphi_{1}(s, \sigma) \mathrm{e}^{\mathrm{ik}(\sigma-s) / \varepsilon} \mathrm{f}_{\mathrm{k}, 1}(\sigma) \mathrm{d} \sigma-\int_{s}^{\infty} \varphi_{2}(s, \sigma) \mathrm{e}^{\mathrm{ik}(\sigma-s) / \varepsilon} \mathrm{f}_{\mathrm{k}, 2}(\sigma) \mathrm{d} \sigma
$$

and

$$
a_{k}^{-}(s)=\int_{ \pm \mathrm{ir}_{\varepsilon}}^{s} \varphi_{1}(s, \sigma) \mathrm{e}^{\mathrm{ik}(\sigma-s) / \varepsilon} \mathrm{f}_{\mathrm{k}, 1}(\sigma) d \sigma+\int_{-\infty}^{s} \varphi_{2}(s, \sigma) e^{\mathrm{ik}(\sigma-s) / \varepsilon} \mathrm{f}_{\mathrm{k}, 2}(\sigma) d \sigma
$$

Here the projections of the $f_{k}$ on the dichotomy subspaces (2.4) are denoted $f_{k, 1}$ and $f_{k, 2}$, and in (5.6) we choose " + " if $k>0$ and " - " if $k<0$.

One now introduces the function spaces $\mathrm{X}^{ \pm}$of $\mathrm{fs}$ with the Sobolev $\mathrm{H}^{1}$ norm in the variable $\theta$ and the sup norm over $S^{ \pm}$in the variable $s$. Also, let $X^{0}$ be the space of $f s$ endowed with the $H^{1}$ norm in $\theta$ and the sup norm with weight $\exp \left[\left(\mathrm{r}_{\varepsilon}-|s|\right) / \varepsilon\right]$ in $s$ over $S^{0}$. We make the assumption that the fundamental solution $\varphi(t, \tau)$ of the linear equation (3.3) has an analytic continuation into the complex strip $S_{\varepsilon}$ in both $t$ and $\tau$ such that the estimates (3.5) and (3.6) hold with $\mathrm{t}$ and $\tau$ on the right hand sides replaced by $\operatorname{Re} t$ and $\operatorname{Re} \tau$. This is verified in our example using the representation (3.7).

Fact 1 Define the ( $\varepsilon$ and $\delta$ dependent $)$ maps

$$
G^{ \pm}: w(s, \theta) \mapsto L^{ \pm} F(w(s, \theta), s, \theta)
$$

where $\mathrm{F}$ and $\mathrm{L}^{ \pm}$are defined in (4.4) and (5.4). Then for each $\varepsilon$ and $\delta, \mathrm{G}^{ \pm}$is a bounded map (maps bounded sets to bounded sets) of $\mathrm{X}^{ \pm}$to itself.

This is proved using Sobolev type estimates; in fact it is useful to break the argument into the two steps of consideration of the maps $w \mapsto F(w, s, \theta)$, to which a standard composition (or $\Omega$ lemma) argument can be applied and a study of the operators $L^{ \pm}$using explicit Fourier series methods. In general, the bound on the image set depends on $\varepsilon$; it could grow as $\varepsilon \rightarrow 0$. To prevent this one needs to balance the growth of the norm of $\mathrm{L}^{ \pm}$and the accumulation of poles in w with the powers of $\varepsilon$ in front of $h$. It is at this stage that some powers of $\varepsilon$ in front of $h$ are needed to get uniformity in $\varepsilon$; this is required for the lower and upper estimates that have the exact distance to the pole in the exponent, and not a smaller one. 
Fact 2 With the assumptions as above, let $\mathrm{B}^{ \pm}$be a bounded subset in $\mathrm{X}^{ \pm}$. Then there exist constants $C_{1}$ and $C_{2}$ depending only on the bounds of the set $B^{ \pm}$such that for any pair of functions $\mathrm{w}^{+}$and $\mathrm{w}^{-}$in $\mathrm{B}^{ \pm}$, we have

$$
\left\|G^{-}\left(w^{-}\right)_{\mid s^{0}}-\left.G^{+}\left(w^{+}\right)\right|_{\mid S^{0}}\right\|^{0} \leq C_{1}\left\|w_{\mid s^{0-}}^{-} w^{+} \mid s^{0}\right\|^{0}+C_{2}
$$

where $\|\cdot\| 0$ is the norm on the space $\mathrm{X}^{0}$.

This is proved by an analysis of the formulas explicitly representing the maps $\mathrm{G}^{+}$and $\mathrm{G}^{-}$. For example, to estimate the difference of the terms coming from the first terms of $(5.6 \mathrm{a}, \mathrm{b})$, we use a Lipschitz property of the composition map $w \mapsto F(w, s, \varepsilon, \theta)$ and this contributes to the first term on the right hand side of (5.8). To estimate the second terms in $(5.6 \mathrm{a}, \mathrm{b})$, for $k>0$, one uses Cauchy's theorem to shift the path of integration in complex $\sigma$-plane along the real axis from $s$ to $\infty$ to a path up the imaginary axis to the point $i r_{\varepsilon}$ and then along the line $\operatorname{Im} \sigma=r_{\varepsilon}$ to $\infty$. Because of the way the extensions to the complex plane have been chosen and the bounds obtained in Fact 1, the integral along the line $\operatorname{Im} \sigma=r_{\varepsilon}$ contributes to the second term on the right hand side (5.8). After subtraction with the corresponding terms in (5.6b), the other terms contribute to the first term on the right side of (5.8).

In the preceding argument, the case $\mathrm{k}=0$ requires special attention. These terms would contribute algebraic, not exponentially small terms, were it not for a crucial cancellation. As above, one first reduces to the case $s=0$ by noting that the difference of the terms contributes to the first term on the right hand side of (5.8). Then we are left to estimate the difference between the terms

$$
\Delta_{1}=\int_{0}^{\infty} \varphi_{2}(0, \sigma) f_{0.2}(\sigma) d \sigma \text { and } \Delta_{2}=-\int_{-\infty}^{0} \varphi_{2}(0, \sigma) f_{0.2}(\sigma) d \sigma
$$

But one checks that we have the symmetry $\Delta_{1}=R \Delta_{2}=\Delta_{2}$, and so these terms cancel. (See the remark below regarding this symmetry assumption).

Now assume that there are bounded neighborhoods $\mathrm{B}^{ \pm}$in $\mathrm{X}^{ \pm}$of 0 which are independent of $\varepsilon$ and $\delta$ and which are mapped into themselves by the $\varepsilon$ and $\delta$ dependent mappings $G^{ \pm}$and so our iterates remain in $B^{ \pm}$for all $n$. This requires an estimate on the poles that occur in the mapping $G^{ \pm}$and the balance between this behaviour and the factors of $\varepsilon$ in front of the nonlinear inhomogeneous term $h$. By choosing $\delta_{0}$ sufficiently small, we can arrange that $C_{1}$ in Fact 2 is less than $1 / 2$. By the contraction mapping principle, the iterates $(n) w^{ \pm}$converge to $w^{ \pm}$in $X^{ \pm}$. For real $s, w^{ \pm}$are related to the stable and unstable manifolds in the following sense: 
Fact 3 For each real $t_{0}$, there are $t_{0}^{t}$ with $\mid t-t_{0}$ of order $\delta$ such that for all real $t$,

$$
u^{ \pm}\left(t, t_{0}, \varepsilon, \delta\right)=\bar{u}_{\varepsilon}\left(t-t_{0}^{t}\right)+\delta w^{ \pm}\left(t-t_{0}^{ \pm}, t / \varepsilon\right)
$$

We prove the required estimates as follows. Making the inductive assumption that $\| \mathrm{w}^{+}$ $-w^{-} \| 0$ is bounded by $2 C_{2}$, the estimate (5.8) shows that the next iterate obeys the same inequality. Passing to the limit, using Fact 3 and rescaling back to the original variables then gives the desired result that the splitting is bounded above by $2 C_{2} \delta \exp \left(-r_{\varepsilon} / \varepsilon\right)$. To get a lower bound, one needs to show that the higher iterates are of lower order than the first iterate. The first term in the iteration is the same as one would get from the Melnikov method, which, in the example can be evaluated explicitly. To estimate the higher order terms requires one to show that the power of $\varepsilon$ in front of $h$ can be used to control the growing norm of the operators $\mathrm{L}^{+}$and $\mathrm{L}^{-}$ as $\varepsilon \rightarrow 0$, and still produce an overall power that increases with each iteration. This is how the condition $p \geq 8$ arises in the example; in that case, we divide $\varepsilon^{8}$ into $\varepsilon^{3}$ to go with $h$ and $\varepsilon^{5}$ to go with $\delta$. These specific powers are chosen to (i) balance the growth in the norm of $L^{ \pm}$as $\varepsilon \rightarrow$ 0 and (ii) to ensurethat the difference between the first and the higher iterates will be small compared to the first iterate. Notice that this analysis is not based on an asymptotic series argument, but rather on a comparison between the first term in the iteration scheme and the subsequent iterates.

Remark 1 From the reversibility assumption, it follows that $\Delta(0, \varepsilon, \delta)=0$ for all $\varepsilon$ and $\delta$. Therefore, the separatrices obviously intersect in this case. However, the proof of the transversality of the intersection requires additional estimates for

$$
\left|\frac{\partial}{\partial t_{0}} \Delta\left(t_{0}, \varepsilon, \delta\right)\right|_{t_{0}=0} \mid
$$

which measures the angle of intersection of the separatrices. Estimates for this again come in two cases, namely upper and lower estimates. These estimates are of the same exponentially small form as those for the spliting distance, with an additional factor of $1 / \varepsilon$. Estimates for the $t_{0}$ derivatives of the iterates in (5.2) can be obtained by the same techniques as for the iterates themselves using the space $\mathrm{H}^{2}$ instead of $\mathrm{H}^{1}$ in the above setting. In the example, again the assumption that $\mathrm{p} \geq 8$ implies that the separatrices do have a transversal intersection with an exponentially small angle of intersection. 
Remark 2 If we consider a one parameter unfolding

$$
\dot{u}=g(u, \varepsilon, \lambda)+\varepsilon^{p} \delta h\left(u, \varepsilon, \frac{t}{\varepsilon}, \lambda\right) \text {, where } \lambda \in R
$$

which agrees with the problem (3.1) for $\lambda=0$, then under a certain non-degeneracy condition with respect to the parameter $\lambda$, a slight modification of our method yields the following result without the reversibility assumption. For sufficiently small $\delta_{0}$, all $t_{0} \in \mathbb{R}$, and all $\varepsilon$ and $\delta$ satisfying $0<\varepsilon \leq 1$ and $0 \leq \delta \leq \delta_{0}$, there exists a value of $\lambda$ given by an expression of the form

$$
\lambda=\lambda_{0}(\varepsilon, \delta)+\lambda_{1}\left(t_{0}, \varepsilon, \delta\right)
$$

such that for this $\lambda$-value, (5.10) has a unique solution which is $\delta_{0}$-close to $\bar{u}_{\varepsilon}\left(t-t_{0}\right)$ (that is, the difference in the sup norm is $\leq$ Const $\cdot \delta_{0}$ ) for all $t \in \mathbb{R}$. In fact, one can find sucessive approximations $\lambda_{n}$ of this $\lambda$-value such that in (4.7)

$$
(n)_{v}+\left(0, t_{0}\right)=(n)_{v}-\left(0, t_{0}\right)
$$

holds for all $n$. Thus it follows that $v^{+}\left(0, t_{0}\right)=v-\left(0, t_{0}\right)$, and the desired solution is given by

$$
\begin{array}{ll}
u\left(s+t_{0}, t_{0}, \varepsilon, \delta\right)=\bar{u}_{\varepsilon}(s)+\delta v^{-}\left(s, t_{0}, \varepsilon, \delta\right) & \text { for all } s \leq 0 \\
u\left(s+t_{0}, t_{0}, \varepsilon, \delta\right)=\bar{u}_{\varepsilon}(s)+\delta v^{+}\left(s, t_{0}, \varepsilon, \delta\right) & \text { for all } s \leq 0
\end{array}
$$

Here $\lambda_{0}(\varepsilon, 0)=0$, and we have the estimate

$$
\left|\lambda_{1}\left(t_{0}, \varepsilon, \delta\right)\right| \leq C \delta \exp \left(-r_{\varepsilon} / \varepsilon\right)
$$

Moreover, near $\lambda=0$, there are no other $\lambda$-values such that (4.10) has a solution which is $\delta_{0^{-}}$ close to $\bar{u}_{\varepsilon}\left(t-t_{0}\right)$ for some $t_{0}$. Thus, if we replace $\delta$ by $\varepsilon$ for example, then there is an exponentially thin wedge-like zone in $(\varepsilon, \lambda)$ - space such that the local stable and unstable manifolds of the perturbed hyperbolic fixed point intersect if and only if $(\varepsilon, \lambda)$ is contained in this zone. Also, the splitting distance is bounded above by $C \varepsilon \exp \left(-r_{\varepsilon} / \varepsilon\right)$ for such values of $\varepsilon$ and 
$\lambda$. This zone of $(\varepsilon, \lambda)$ - values corresponds to the Amold tongues of perturbed periodic solutions (cf. Scheurle [1986]).

Our techniques also show that $\Delta\left(t_{0}, \varepsilon, \delta\right)$ in $(5.1)$ is bounded above by $C \delta \exp \left(-r_{\varepsilon} / \varepsilon\right)$ as $\varepsilon \rightarrow 0$ whenever the stable and the unstable manifolds of the perturbed hyperbolic fixed point of (3.1) intersect in a solution which is $\delta_{0}$-close to $\bar{u}_{\varepsilon}\left(t-t_{0}\right)$ with some $t_{0}=t_{0}^{*}(\varepsilon, \delta)$ for all small $\varepsilon$. Besides reversible problems, where $t_{0}^{*}(\varepsilon, \delta)=0$ for all $\varepsilon$, Hamiltonian systems also have this property (cf. Amold [1965]). We point out, however, that the equation that we have considered as an example is locally, but not globally Hamiltonian. Our theory requires a homoclinic orbit, so we have chosen the phase space to be the cylinder.

\section{A 2:1 Resonance and KAM Theory}

In KAM theory, arguments based on numerical evidence and formal calculations lead to the conjecture that one has exponentially fine splittings and exponentially long escape times. Some rigorous but rough upper bounds for this phenomena have been given by Nekhoroshev [1971, 77] and Neishtadt [1984]; see also the discussion in Amold [1978, pp. 395ff and 407], Chirikov [1979], and Simo and Fontich [1985]. The analyticity argument of Cushman [1978] and Koslov [1984] (and reference therein) uses the Poincaré-Melnikov method to prove that the separatrices do split for most parameter values. However, it is not easy to prove from these arguments that splittings really do occur for specific parameter values and what the sharp upper and lower estimates for the splitting distances are. The seriousness and significance of this difficulty was emphasized by Sanders [1982].

Exponentially fine phenomena appear to be prevalent in a number of situations beyond those discussed here and in the next section. For example:

1 The action appears to change by an exponentially small amount in adiabatic theory (see, for example, Lenard [1959], Meyer [1976], and Berry [1985] - see also Marsden, Montgomery and Ratiu [1988]). We expect that our techniques will be relevant for these problems.

2 The existence of breathers in the $\phi^{4}$ model involves exponentially small phenomena (see Segur and Kruskal [1987]).

3 The growth of dendritic crystals also involves exponentially small phenomena (see Kruskal and Segur [1987]).

4 Various problems in critical phenomena in water waves also seem to involve these issues; $\mathrm{cf}$. Hunter and Scheurle [1987]. 
5 Exponentially small phenomena are known to occur in the study of relaxation oscillations; cf., Eckhaus [1982]

6 Finally, it has been suspected for some time that these problems also arise in the unfolding of degenerate singularities; see for example, Takens [1974]. We shall illustrate the basic ideas in .87 .

Here we consider a simple illustration of why these problems come up in KAM theory. Consider the dynamics of two coupled oscillators with Hamiltonian, written in action angle variables, of the form

$$
\mathcal{H}(\theta, \mathrm{I}, \phi, \mathrm{J}, \varepsilon)=\mathrm{F}(\mathrm{I})+\mathrm{J}+\varepsilon \mathrm{K}(\theta, \mathrm{I}, \phi)
$$

We have taken the second oscillator to be a harmonic oscillator and the coupling independent of $J$ purely for simplicity. If we set $H=$ constant, (3.1) determines $J$. We can also let $\phi=t$ be the new time, so (3.1) becomes equivalent to a forced one degree of freedom system with Hamiltonian

$$
H(\theta, I, t, \varepsilon)=F(I)+\varepsilon K(\theta, I, t)
$$

For example, choose $K(\theta, I, \phi)=I \sin ^{2} \theta \cos \phi$ and $F(I)=I-I^{2} / 2$. Then one sees that the circle $I=1 / 4$ resonates with the forcing in a 2:1 resonance. To study it, we make the change of variables

$$
I=\frac{1}{4} \cdot \sqrt{\varepsilon} p, \quad \theta=\frac{t}{2}+\Psi
$$

to get

$$
\begin{gathered}
\dot{\psi}=\sqrt{\varepsilon}(2 \mathrm{p})+\varepsilon\left[\frac{1}{4} \cos 2 \psi+\cos 2(\psi+\mathrm{t})-\frac{1}{2} \cos \mathrm{t}\right], \\
\dot{\mathrm{p}}=\sqrt{\varepsilon}\left[-\frac{1}{8} \sin 2 \psi+\sin 2(\psi+\mathrm{t})\right]+\varepsilon\left[\frac{\mathrm{p}}{2} \sin 2 \psi+\sin 2(\psi+\mathrm{t})\right]
\end{gathered}
$$

Now one removes the t-dependence at order $\sqrt{\varepsilon}$ by the averaging transformation

$$
\psi=\psi^{\prime}, \quad p=p^{\prime}-\frac{\sqrt{\varepsilon}}{16} \cos 2\left(\psi^{\prime}+t\right)
$$


Dropping the primes, the new system becomes Hamiltonian with

$$
H=\sqrt{\varepsilon} F(\psi, p)+\varepsilon G(\psi, p, t)
$$

where

$$
F(\psi, p)=p^{2}-\frac{1}{16} \cos 2 \psi,
$$

and

$$
G(\psi, p, t, \varepsilon)=\frac{p}{8}[2 \cos 2 \psi+3 \cos 2(\psi+t)-4 \cos t]+O\left(\varepsilon^{1 / 2}\right)
$$

Rescaling time to $\tau=\sqrt{\varepsilon} t,(6.6)$ transforms to

$$
H=F(\psi, p)+\sqrt{\varepsilon} G\left(\psi, p, \frac{\tau}{\sqrt{\varepsilon}}\right)
$$

which has our form of a rapidly forced perturabation of the Hamiltonian F, which has homoclinic orbits with

$$
p= \pm \frac{1}{2 \sqrt{2}} \operatorname{sech}(4 \sqrt{2} \tau)
$$

The situation is shown in Figure 5, represented in $(\mathrm{I}, \theta)$ coordinates, viewed as polar coordinates. With the addition of $\sqrt{\varepsilon} \mathrm{G}(\psi, p, t / \sqrt{\varepsilon})$, one develops stochastic layers around the homoclinic orbits shown in figure 5. 

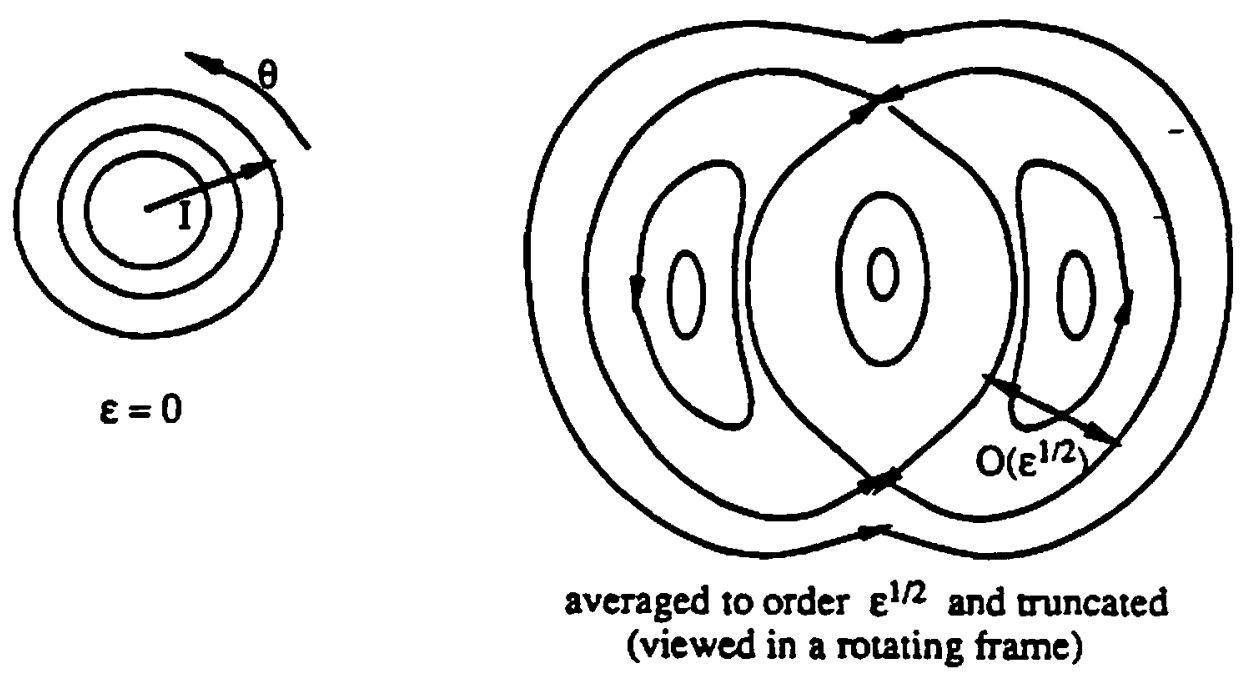

Figure 5

A formal Melnikov calculation suggests the splitting distance is of order

$$
\frac{\pi}{256 \sqrt{\varepsilon}} \operatorname{sech}\left(\frac{\pi}{8 \sqrt{2 \varepsilon}}\right)
$$

which is exponentially small. Our upper estimate shows that

$$
\text { spliting } \leq c \sqrt{\varepsilon} \exp \left(-\left(\frac{\pi}{8 \sqrt{2}}-\eta\right) \frac{1}{\sqrt{\varepsilon}}\right)
$$

with a similar estimate for the splitting angle. Note that (6.10) is compatible with (6.9), although (6.9) suggests a sharper result.

Our lower estimates do not apply to (6.7) since the same power of $\varepsilon$ appears as an amplitude coefficient in front of $G$ and also as the denominator of $\tau / \sqrt{\varepsilon}$. Our analysis of the estimates suggests that it may be very difficult to rigorously establish an estimate above and below by an expression like (6.9). However, one can show the following: Consider the same system with an additional term:

$$
H=I-\frac{I^{2}}{2}+J+\varepsilon I \sin ^{2} \theta \cos \phi+\varepsilon^{2} H_{2}
$$


We choose $\mathrm{H}_{2}$ such that after averaging, terms of lower order in $\varepsilon$ cancel, leaving only higher order terms in $\varepsilon$ and our lower bound now does apply. The algebra involved to get $\mathrm{H}_{2}$ is a little involved, so we illustrate the result with a simpler explicit example. Consider

$$
\ddot{\phi}+\sin \phi=\varepsilon \sin \left(\frac{t}{\varepsilon}\right) \text {. }
$$

Again, the upper bound $C \varepsilon e^{-(\pi / 2-\eta) / \varepsilon}$ is valid, but an optimal upper and lower estimate are not known. However, we can modify (6.12) a bit to

$$
\ddot{\phi}+\sin \phi=\varepsilon \sin \left(\frac{t}{\varepsilon}\right)+\varepsilon^{2} h\left(\frac{t}{\varepsilon}, \varepsilon, \phi\right),
$$

where

$$
h(\tau, \varepsilon, \varphi)=\varepsilon \cos \varphi \sin \tau+\frac{\varepsilon^{4}}{2} \sin \varphi \sin ^{2} \tau-\frac{\varepsilon^{7}}{3} \cos \varphi \sin ^{3} \tau
$$

so that (6.13) satisfies

$$
h^{C_{2} \varepsilon^{12} e^{-\pi / 2 \varepsilon} \leq \text { splitting } \leq C_{1} \varepsilon^{12} \varepsilon^{-\pi / 2 \varepsilon} .}
$$

This is done by choosing K so that after averaging, the system has the form required for both our upper and lower estimates.

Thus, while we cannot prove the upper and lower estimates for (6.12), there is a nearby system (6.13) for which they are valid. We conclude that while the upper estimates are fairly robust, the lower estimates appear to be very delicate and in fact one can perturb a given system slightly to get a splitting distance (and angle) much smaller than one might have expected -- see the extra power of $\varepsilon^{12}$ in (6.14). Even more extreme, one can sometimes add a term which completely cancels all the higher order terms and the perturbed system becomes completely integrable! For instance, a trivial example of this sort is the completely integrable system

$$
\begin{aligned}
& \dot{x}=y-\varepsilon^{2} \sin \left(\frac{t}{\varepsilon}\right) \\
& \dot{y}=\sin x+\varepsilon \cos \left(\frac{t}{\varepsilon}\right)
\end{aligned}
$$


which is simply a complicated way of writing the pendulum equation. This general behavior appears to be rather common and shows that an asymptotic estimate good enough to give lower bounds independent of (or robust with respect to) all higher order terms is not possible.

\section{Exponentially Small Splittings in a Bifurcation Problem}

We consider the problem of a Hamiltonian saddle node bifurcation

$$
\ddot{x}+\mu x+x^{2}=0
$$

with the addition of higher order terms and forcing:

$$
\ddot{x}+\mu x+x^{2}+\text { h.o.t. }=\delta f(t)
$$

The phase portrait of (7.1) is shown in Figure 6.

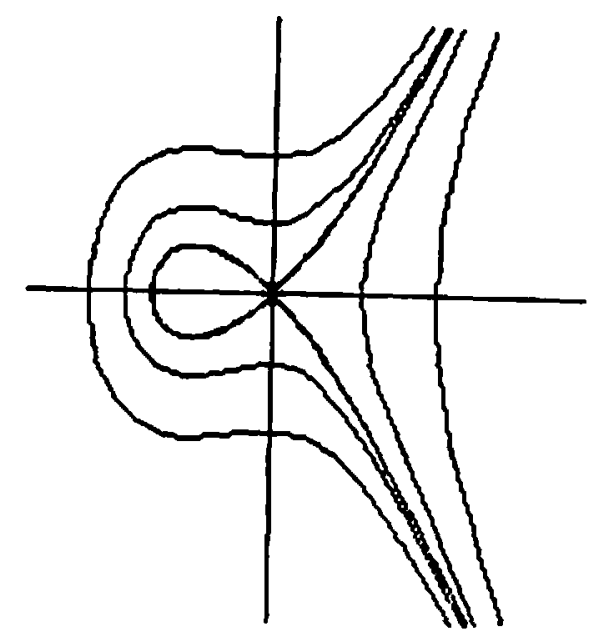

$\mu<0$

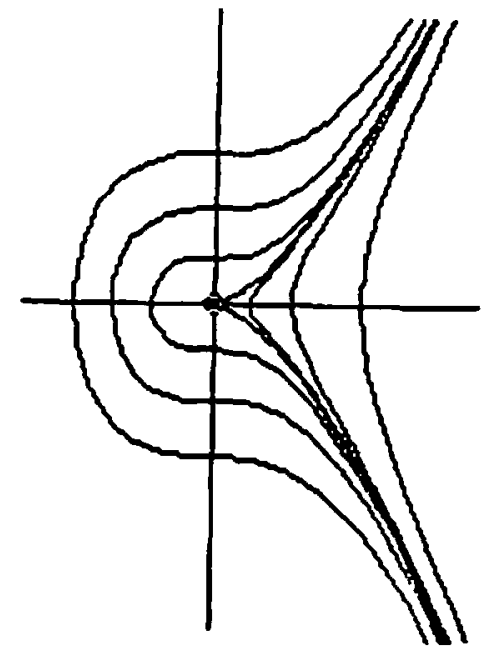

$\mu=0$

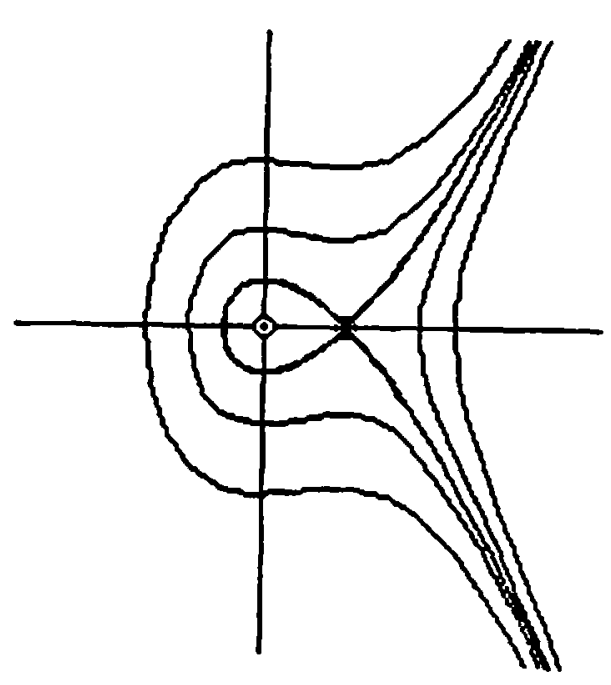

$\mu>0$

Figure 6 
Exponentially Small Splittings

27

The system (7.1) is Hamiltonian with

$$
H(x, p)=\frac{1}{2} p^{2}+\frac{1}{2} \mu x^{2}+\frac{1}{3} x^{3}
$$

Let us first consider the system without higher order terms:

$$
\ddot{x}+\mu x+x^{2}=\delta f(t)
$$

To study it, we rescale to blow up the singularity:

$$
x(t)=\lambda \xi(\tau)
$$

where $\lambda=|\mu|$ and $\tau=t \sqrt{\lambda}$. We get

$$
\begin{gathered}
\ddot{\xi}-\xi+\xi^{2}=\frac{\delta}{\mu^{2}} f\left(\frac{\tau}{\sqrt{-\mu}}\right), \quad \mu<0, \\
\ddot{\xi}+\xi+\xi^{2}=\frac{\delta}{\mu^{2}} f\left(\frac{\tau}{\sqrt{\mu}}\right), \mu>0 .
\end{gathered}
$$

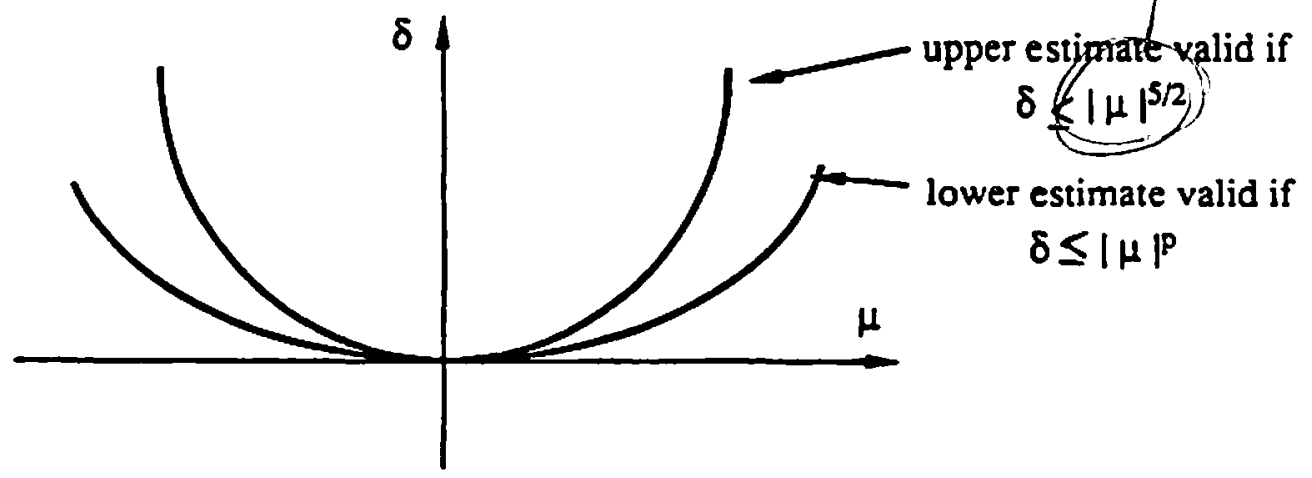

Figure 7 
Our exponentially small estimates apply to (7.6a and b). We will get upper and lower estimates in algebraic sectors of the $\delta-\mu$ plane, as in Figure 7. The power $\underline{p}$ depends on the nature of $f$.

Now we consider

$$
\ddot{x}+\mu x+x^{2}+x^{3}=\delta f(t)
$$

With $\delta=0$, there are equilibria at

$$
\begin{aligned}
& x=0,-r, \text { or }-\frac{\mu}{r} \\
& \dot{x}=0
\end{aligned}
$$

where

$$
\mathrm{r}=\frac{1+\sqrt{1-4 \mu}}{2}
$$

which is approximately 1 when $\mu=0$. The phase portrait of equation (7.7) with $\delta=0$ and $\mu=$ $-\frac{1}{2}$ is shown in Figure 8. As $\mu$ passes through 0 , the small lobe in Figure 8 undergoes the same bifurcation as in Figure 5, with the large lobe changing only slightly.

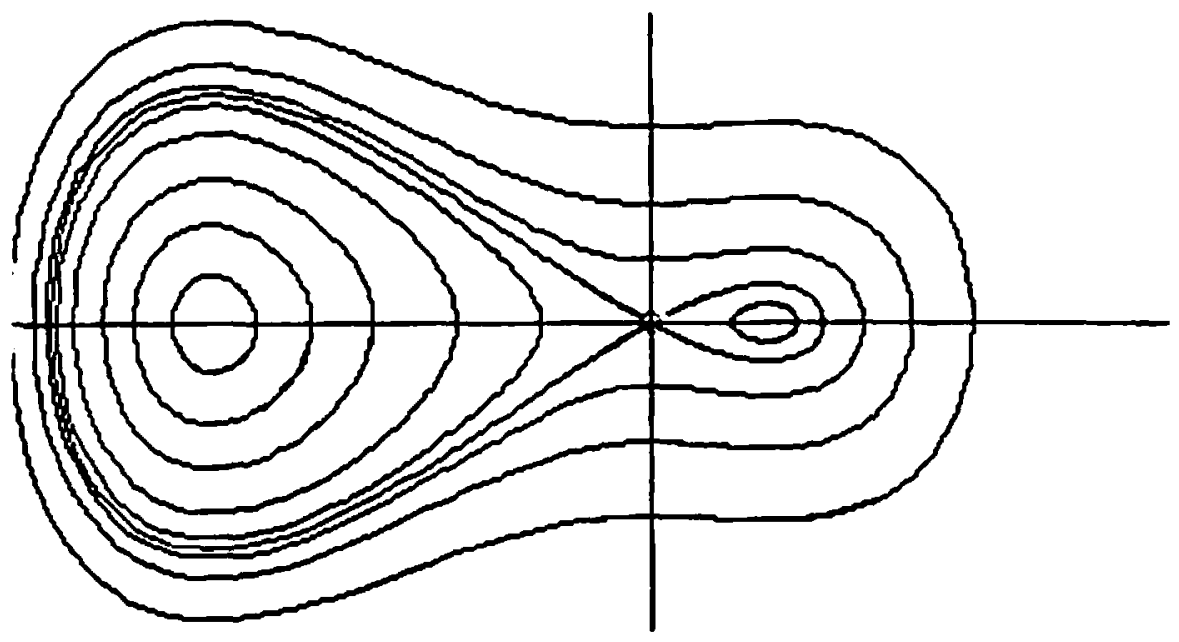

Figure 8 
Again we rescale by (7.5) to give

$$
\begin{aligned}
& \ddot{\xi}-\xi+\xi^{2}-\mu \xi^{3}=\frac{\delta}{\mu^{2}} \mathrm{f}\left(\frac{\tau}{\sqrt{-\mu}}\right), \mu<0, \\
& \ddot{\xi}+\xi+\xi^{2}+\mu \xi^{3}=\frac{\delta}{\mu^{2}} \mathrm{f}\left(\frac{\tau}{\sqrt{\mu}}\right), \mu>0 .
\end{aligned}
$$

Notice that for $\delta=0$, the phase portrait is $\mu$-dependent. The homoclinic orbit surrounding the small lobe for $\mu<0$ is given explicitly in terms of $\xi$ by

$$
\xi(\tau)=\frac{4 e^{\tau}}{\left(e^{\tau}+\frac{2}{3}\right)^{2}-2 \mu},
$$

which is $\mu$-dependent. An interesting technicality is that without the cubic term, we get $\mu$ independent double poles at $\tau= \pm i \pi+\log 2-\log 3$ in the complex $\tau$-plane, while (7.10) has a pair of simple poles that splits these double poles to the pairs of simple poles at

$$
\tau= \pm i \pi+\log \left(\frac{2}{3} \pm i \sqrt{2 \lambda}\right)
$$

where again $\lambda=|\mu|$. (There is no particular significance to the real part, such as $\log 2-\log 3$ in the case of no cubic term, since this can always be gotten rid of by a shift in the base point $\xi(0)$.)

If a quartic term $x^{4}$ is added, these pairs of simple poles will split into quartets of branch points and so on. Thus, while the analysis of higher order terms has this interesting $\mu$ dependence, it seems that the basic exponential part of the estimates,

$$
\exp \left(-\frac{\pi}{\sqrt{|\mu|}}\right),
$$

remains intact. 
Discussion and Conclusions We have given conditions under which one can obtain transversal intersection and both upper and lower estimates for the angle of intersection and for the splitting distance of separatrices in a rapidly forced system with a homoclinic orbit; the bounds obtained are exponentially small in the frequency parameter. Our main example is the rapidly forced pendulum equation, which is related to the pendulum suspended from a very stiff elastic rod. This example is a nonautonomous conservative system with a homoclinic orbit. With the addition of damping, exponentially small splitting and intersections of the separatrices typically occur only in an exponentially small wedge in parameter space (see Remark 2 above). Exponentially small splittings also occur in the unfolding of degenerate singularities and in KAM theory as was discussed in the last two sections. In future work we shall be applying these ideas to other problems, including sharp exponential estimates for adiabatic invariants of the sort that occur in Berry's phase.

\section{References}

V. I. Arnold [1965] Sur une propriétè topologique des applications globelement canonique de la méchanique classique. C. R. Acad. Sci. Paris. 26, 3719-3722.

V. I. Arnold [1978] Mathematical Methods of Classical Mechanics, Graduate Texts in Mathematics 60, Springer-Verlag, NY.

M. Berry [1985] Classical Adiabatic Angles and Quantal Adiabatic Phase, J. Phys. A: Math. Gen. 18, 15-27

T. Bountis, V. Papageorgiou, and M. Bier [1986] On the singularity analysis of intersecting separatrices in near-integrable dynamical systems, Physica $D$ (to appear).

B. Chirikov [1979] A universal instability of many dimensional oscillator systems, Phys. Reports, 52, 265.

R. Cushman [1978] Examples of non-integrable analytic Hamiltonian vector fields with no small divisors, Trans. Am. Math. Soc. 238, 45-55.

G. Dangelmayr and E. Knobloch [1987] On the Hopf Bifurcation with Broken O(2) Symmetry, preprint.

W. Eckhaus [1982] Relaxation Oscillations, Including a Standard Chase on French Ducks, in Asymptotic Analysis II, ed. by F. Verhulst, Springer Lecture Notes in Mathematics, 985, 449 494.

M. Golubitsky and I. Stuart [1986] Symmetry and stability in Taylor Couetre flow, SIAM J. Math. Anal. 17, 249-288. 
J. Guckenheimer and P.J. Holmes [1983] Nonlinear oscillations, dynamical systems, and the bifurcation of vector fields, Applied Mathematical Sciences, Springer-Verlag, N.Y.

P. Hartman [1982] Ordinary Differential Equations, Wiley [1964], Second Edition, Birkhäuser.

PJ. Holmes and J.E. Marsden [1981] A partial differential equation with infinitely many periodic orbits: Chaotic oscillations of a forced beam, Arch. for Rational Mech. and Analysis 76, 135166.

P.J. Holmes and J.E. Marsden [1982a] Horseshoes in perturbations of Hamiltonian systems with two degrees of freedom, Commun. Math. Physics 82, 523-544.

P.J. Holmes and J.E. Marsden [1982b] Melnikov's method and Amold diffusion for perturbations of integrable Hamiltonian systems, J. Math. Phys. 23, 669-675.

P.J. Holmes and J.E. Marsden [1983] Horseshoes and Amold Diffusion for Hamiltonian Systems on Lie Groups, Indiana Univ. Math. J. 32, 273-310.

P.J. Holmes, J.E. Marsden, and J. Scheurle [1988] Exponentially Small Splitting of Separatrices, Proc. Nat. Acad. Sci. (to appear).

J. Hunter and J. Scheurle [1987] preprint

V.V. Koslov [1984] Integrability and non-integrability in Hamiltonian mechanics, Russian Math. Surveys, 38, 1-76.

M. Kruskal and H. Segur [1987] Asymptotics beyond all orders in a model of dendritic crystals, preprint.

V. Lazutkin, I.G. Shachmanski and M.B. Tabanov [1987], Separatrix splitting for the standard and semi-standard mappings, preprint.

A. Lenard [1959] Adiabatic Invariance to all Orders, Ann. of Phys. 6, 261-276

J. Marsden, R. Montgomery and T. Ratiu [1988], Reduction, Symmetry, and Berry's Phase in Classical Machanics: Theory preprins.

V.K. Melnikov [1963] On the Stability of the Center for Time Periodic Perturbations, Trans. Moscow Math. Soc. 12, 1-57

R.E. Meyer [1976] Adiabatic Variation - Part V: Nonliear Near-Periodic Oscillator, J. Appl. Math. and Physics (ZAMP), 27, 181-195.

R.E. Meyer [1982] Wave Reflection and Quasiresonance, in Theory and Application of Singular Perturbations, W. Eckhaus and E. M. de Jager, eds., Springer Lecture Notes in Mathematics, 942, 84-112.

N.N. Nekhoroshev [1971] The behavior of hamiltonian systems that are close to integrable ones, Funct. An. Appl. 5, 4-10.

N.N. Nekhoroshev [1977] An exponential estimate of the time of stability of nearly-integrable Hamiltonian systems, Russian Math. Surveys 32, 1-65. 
A. Neishtadt [1984] The separation of motions in systems with rapidly rotating phase, P.M.M. USSR, 48, 133-139.

H. Poincaré [1890] Sur la problème des trois corps et les équations de la dynamique, Acta Math. 13, 1-271

P. van Moerbeke [1983] Algebraic complete integrability of Hamiltonian systems and Kac-Moody Lie algebras, Proc. Intern. Cong. Mathem. 881-899.

V. K. Melnikov [1963] On the stability of the center for time periodic perturbations, Trans . Moscow Math. Soc. 12, 1-57.

J.A. Sanders [1982] Melnikov's method and averaging, Celestial Mechanics, 28, 171-181.

J. Scheurle [1987] Asymptotic properties of Amold tongues. CMS Conference Series (AMS Publications) 8, 655-663

J. Scheurle and J. Marsden [1984] Bifurcation to quasi-periodic tori in the interaction of steady state and Hopf bifurcations, SIAM J. Math. Anal. 15, 1055-1074.

H. Segur and M. Kruskal [1987] Non-existence of Small-Amplitude Breather Solutions in $\varphi^{4}$ Theory. Phys. Rev. Lett. 58, 747-750

C. Simo and E. Fontich [1985] On the smallness of the angle between split separatrices, Proc. Colloq. sur Geom. Symplectique et Mechanique, Montpellier.

F. Takens [1974] Forced Oscillations and Bifurcations, Comm. Math. Inst.., Rijkuniversiteit Utrecht, 3, 1-59.

E. Zehnder [1973] Homoclinic points neas Elliptic fixed points, Comm. Pure and Applied Math. 26, 131-182.

S. L. Ziglin [1982] Branching of solutions and nonexistence of first integrals in Hamiltonian mechanics, Finct. Anal. Appl. 16, 30-41, 17, 8-23.

Philip Holmes

Theoretical and Applied Mechanics and Mathematics

Comell University

Ithaca, N.Y. 14853

Jerrold Marsden

Department of Mathematics

University of California

Berkeley, California, 94720

Jurgen Scheurle Institüt für Angew. Math.

Universitat Hamburg

Bundesstrasse 55

D-2000 Hamburg 13

West Germany and

Department of Mathematics

Cornell Universtiy

Ithaca, N.Y. 14853-7901

and

Department of Mathematics

Colorado State University

Fort Collins, CO 80523 